Revue d'histoire de l'Amérique française

REVUE D.HISTOIRE DE L'AMÉRIQUE FRANÇAISE

\title{
Mgr Adélard Langevin d'après une partie de sa correspondance
}

\section{Lionel Groulx}

Volume 1, numéro 4, mars 1948

URI : https://id.erudit.org/iderudit/801415ar

DOI : https://doi.org/10.7202/801415ar

Aller au sommaire du numéro

Éditeur(s)

Institut d'histoire de l'Amérique française

ISSN

0035-2357 (imprimé)

1492-1383 (numérique)

Découvrir la revue

Citer cet article

Groulx, L. (1948). Mgr Adélard Langevin d'après une partie de sa

correspondance. Revue d'histoire de l'Amérique française, 1(4), 569-594.

https://doi.org/10.7202/801415ar d'utilisation que vous pouvez consulter en ligne.

https://apropos.erudit.org/fr/usagers/politique-dutilisation/ 


\section{MGR ADÉLARD LANGEVIN D'APRĖS UNE PARTIE DE SA CORRESPONDANCE}

Qu'on ne cherche, en ces pages, - le titre le dit assez - ni une biographie, ni un portrait en pied du célèbre archevêque. Ce sera un Mgr Langevin, tel qu'apparu dans une portion précise de sa correspondance; ou, si l'on prefère, quelques traits de cette énergique et séduisante figure révélés par une soixantaine de lettres échangées avec un ami laïc.

Quelle valeur historique ou illustrative accorder tout d'abord a cette correspondance de l'archevêque? Comme tout document, le document épistolaire n'est recevable, en histoire, que soumis à la loupe de la critique. La lettre n'en revêt pas moins une valeur documentaire supérieure, par exemple, à celle du journal intime. Elle procède de plus de spontanéité, tient davantage de l'improvisation. L'épistolier ne se présente point à nous, dans l'ordinaire, sous l'image classique du rédacteur de journal ou de mémoires, que ce soit d' " Outre-tombe " ou d' (Avant-tombe ", écrivain en manchettes, assis devant une large glace qui lui renvoie son personnage et livré ainsi, même à son insu, aux tentations du plus périlleux narcissisme.

Sans doute, faut-il encore distinguer entre les variétés du genre épistolaire. On n'utilisera qu'avec une extrême discrétion, la lettre officielle ou diplomatique dont le contenu est souvent fait de ce qui ne s'y trouve point. D'aussi pauvre valeur documentaire en histoire, la lettre du bel esprit trop averti des itinéraires de sa littérature, promenée de main en main, parmi les amis, dans les salons, épistoliers qui écrivent, comme dirait Chateaubriand, "pour l'immortalité ", et à qui il ne manque, pour y parvenir, que le talent de la coquette marquise. Permettons-nous encore une distinction entre la lettre dictée et la lettre autographe: la première, d'un style plus surveillé et d'ailleurs relue et retouchée avant d'être signée; la seconde, jetée tout d'un jet sur le papier, expression plus immédiate, plus vivante de la pensée. 
La critique doit aussi compter, et cela aussi va de soi, avec la personnalité et l'état de vie des correspondants. Des personnages officiels, des gens plus ou moins étrangers l'un à l'autre, ne s'écrivent point avec l'abandon de parents ou d'amis intimes. Et même, parmi les amis, les catégories s'établissent. Il y a ceux à qui l'on écrit peu souvent et à qui l'on ménage les confidences; et il y a les confidents à qui l'on ne cache rien et qui deviennent ainsi les témoins et les interprètes les plus authentiques d'une existence.

Ces prémisses posées, voici nos deux correspondants. L'Archevêque de Saint-Boniface, qui porte le nom d'Adélard Langevin, est bien connu dans l'histoire. Il est né le 23 août 1855 à Saint-Isidore de Laprairie, dans la province de Québec. Oblat de Marie-Immaculee, il quitte, en 1892, à l'âge de trente-sept ans, l'Université d'Ottawa pour s'en aller dans l'Ouest, au Manitoba. Ses supérieurs le destinent apparemment à la succession de Mgr Taché. Le vieil archevêque lui confiera, avant sa mort: "Je vous désirais depuis dix ans " ". Attaché, à son arrivée là-bas, à la cure de Sainte-Marje de Winnipeg (St Mary's Church), vicaire de missions, puis, tout en restant vicaire de missions, curé de Sainte-Marie en 1894, il devient le 8 janvier 1895, successeur de Mgr Taché. Le jeune archevêque charge ses épaules d'une terrible succession. Il hérite de la querelle scolaire manitobaine, la plus grave de toutes celles qui ont remué au Canada les passions nationales et religieuses; et il entrait, dans la querelle, en 1895, à sa phase la plus aiguë. La question des écoles du Manitoba et du Nord-Ouest avait surgi, au surplus, au lendemain de l'affaire Riel qui venait de chauffer à blanc l'antagonisme des races. Nantie d'une position constitutionnelle qu'on aurait pu croire inexpugnable en pays de droit chrétien, la minorité catholique et française du Manitoba se défendit avec énergie. Il y allait de l'avenir de l'Église dans les prairies. Dès les premiers assauts, l'archevêque Taché, vénérable vieillard, vétéran des missions de l'ouest canadien, se trouva le chef naturel des persécutés. Son jeune successeur ne pouvait se dérober à ce même rôle. Il ne semble pas, du reste, qu'il en ait eu l'envie. Mais l'on imagine facilement le choc qu'il éprouve. Il est fils de l'est, du Québec pacifique et somnolent. Il vient même de respirer quelque temps l'air glacé de la capitale fédérale. De but en blanc, voici cet homme au

1. Mgr Langevin, Mandement de prise de possession, p. 9. 
cœur ardent, à l'âme de feu, jeté dans une lutte d'une extrême violence, obligé d'évoluer sur le terrain juridique et politique, dans un débat fatalement épineux pour un homme d'Église.

Le correspondant de l'archevêque est le colonel Alphonse Audet. Montréalais de naissance, âgé en 1892, de 52 ans, ce colonel, tout galonné et flamboyant qu'il soit, n'en est pas un tout à fait de parade. Dans ses états de services, il compte, à tout le moins, les opérations de police de 1866 et de 1870 contre les Féniens. Mais il a surtout livré des batailles politiques. Quelque temps rédacteur en chef de la Minerve, puis traducteur en chef des débats de la Chambre des Communes à Ottawa, puis directeur des Archives à la Secrétairerie d'État, organisateur aussi du parti conservateur dans le district de Montréal, il a été, un peu pendant tout ce temps, conseiller politique d'Adolphe Chapleau. ${ }^{2}$ Nous avons donc affaire à un personnage considérable du monde conservateur. Homme de parti, sans conteste, mais que l'esprit de parti n'a pas envoûté. Il se fâchera tout de bon le jour où l'archevêque malmènera trop rudement ses chefs politiques. Il ne se cache point pour autant la dégénérescence d'un entourage qui paralyse ces mêmes chefs, eux mêmes d'ailleurs atteints, comme tout le parti, de gangrène sénile. Le poison classique en démocratie parlementaire, l'usure du pouvoir, a fait ici son ouvre. A travers la correspondance, on voit le pauvre colonel suer sang et eau à la recherche de remèdes et de réformes qui pourraient galvaniser le moribond. Très honnête homme, d'esprit élevé et cultivé, Alphonse Audet a tôt discerné les graves enjeux de la querelle manitobaine. Seules, il le confessera à son ami l'archevêque, les Ecoles - entendez l'espoir d'obtenir un règlement équitable - le "retiennent au parti " ${ }^{3}$. Voyons donc en lui un de ces bons bleus de l'époque, gens probes et candides, qui auraient donné leur âme pour le salut du parti idolâtré, et qui, tout en assistant navrés au naufrage du bateau, se morfondaient en projets de renflouement.

Sans se tutoyer, les deux hommes sont des intimes. Entre eux l'amitié est née, prompte et vive, comme entre esprits pourvus d'antennes l'un pour l'autre. La correspondance s'ouvre par une lettre du

2. Ces notes sont extraites d'une biographie inédite du Colonel Audet par F.-J. AUDET.

3. Lettre du 14 fév. 1896. 
Père Langevin, écrite de l'Université d'Ottawa, ou plus exactement de "University of Ottawa ", comme on disait et écrivait en ce tempslà, et datée du 17 nov. 1892, lettre banale pour simple recommandation d'un protégé. La lettre est adressée à " Monsieur le Colonel ». Quatre jours plus tard une deuxième lettre s'adresse cette fois à " Monsieur et cher colonel ». L'année suivante le Père partait pour Winnipeg. Le Colonel ne sera plus maintenant que " Mon cher Colonel ») et " Mon bien cher Colonel ). Dans sa première lettre datée du Manitoba (6 juillet 1893) le Père fait cette confidence à son correspondant: " Je me demande comment nous en sommes venus à nous comprendre si facilement $)$. Un an plus tard, 6 juillet 1894, autre confidence encore plus affectueuse: "Ai-je jamais rencontré un ami plus sincère. Je me demande comment j'ai pu obtenir cette grâce ". Une amitié vraie, profonde, lie donc ces deux hommes. On le perçoit au ton, à l'abandon des propos et aussi à quelques finales des lettres: " Je suis à vous de tout cœur "; " Ex præcordüs tuis 》; " Bien à vous in toto corde " "; " Toujours et de plus en plus votre ami de cœur " ${ }^{5}$; " Je vous bénis comme on bénit un ami de cœur $)^{6}$. En 1900 un malentendu se glisse entre les correspondants; navré l'archevêque se laisse aller à cet aveu mélancolique:

( J'ai beaucoup souffert depuis notre entrevue, et c'est votre silence qui lui a donné une gravité que je ne lui attribuais pas d'abord... Ai-je besoin de vous dire que j'oublie tout dès que j'apprends que vous n'êtes pas perdu pour moi! L'autre jour encore je retrouvais plusieurs de vos lettres et j'étais en proie à une grande tristesse. "C'est ainsi que la vie s'effeuille! " ai-je dit. ") "

Le colonel devient, pour son ami de Saint-Boniface, un correspondant précieux. Il lui fournit documents officiels et journaux de l'est. C'est, pour une part, documenté par le colonel, que Mgr Taché pourra écrire quelques-unes de ses retentissantes brochures sur la question des écoles du Manitoba. Le Colonel sert d'agent de liaison entre les autorités politiques d'Ottawa et l'archevêché de Saint-Boni-

4. Lettre du 19 aout 1893.

5. Lettre du 20 oct. 1893.

6. Lettre du 25 nov. 1895

7. Lettre du 16 juin 1900. 
face. Il secoue la torpeur des hommes de la capitale, des ministres canadiens-français désemparés devant l'imbroglio scolaire. Esprit plutôt grave et calme, en contraste avec son bouillant ami, il peut se permettre de le conseiller, et même de le relever aux heures d'abattement. En ces circonstancès, le Colonel s'improvise historien-philosophe. Il sert à l'archevêque, en de longues dissertations, la philosophie de l'histoire canadienne. De ces épîtres du Colonel, nous $n \in$ pouvons malheureusement juger que par les réactions ou réponses de son correspondant. Nous ne possédons pas ses lettres. Aux archives de l'archevêché de Saint-Boniface, on les dit introuvables. Il semble, du reste, que Mgr Langevin ait renvoyé au Colonel une partie au moins de sa correspondance. Il lui écrit, le 30 octobre 1899: "J'ai oublié de vous demander si vous aviez reçu vos lettres qui prennent l'importance d'un document historique très précieux ").

Arrêtons là les données indispensables à l'étude de cette liasse de lettres de Mgr Langevin. Le document est de valeur. Il y a chance que l'on y puisse saisir le personnage sur le vif, l'homme dans la nudité de ses sentiments, dans sa pensée toute chaude.

L'homme, c'est déjà lui qui nous est apparu dans les extraits de ses lettres cités plus haut: un homme que le caractère épiscopal n'a ni tué, ni diminué, mais qu'on trouve capable d'une grande amitié, homme d'une sensibilité virile et fine. Et je n'entends pas seulement cette sorte de sensibilité mi-physique, mi-intellectuelle, qu'on dit propre aux orateurs, dispcsitions émotives mises en acte par le simple mécanisme de la parole, le contact d'un auditoire, et qui firent de l'évêque Langevin un homme si remarquablement éloquent. L'orateur, en effet, on le trouve presque en chacune de ses lettres, dans le tour rif de la pensée, de la phrase, dans le choc des figures et des mots, dans l'émotion presque toujours sous-jacente. Mais je veux parler aussi d'une autre forme de sensibilité, de celle qui jaillit de toutes les profondeurs de l'être noble, qu'on dirait faite de la passion des idées et des grandeurs morales, qui rend capable d'enthousiasme et prompt, en même temps, aux révoltes intérieures, aux indignations superbes devant tes mensonges ou les petitesses de la vie. 
Sensibilité d'élite qui pourrait bien être mère ou sœur de la fierté, vertu des consciences saines, élevées.

Fierté, autre trait de cet homme, trait peut-être dominant, mis en vigoureux relief par la correspondance. Fierté d'homme libre, fierté de citoyen et de chrétien, passionnément féru de son droit, de l'égalité de tous devant la loi, devant la constitution du pays; fierté séduisante de chevalier qui, devant la bassesse, l'injustice, l'arrogance des forts, atteint facilement au panache. Fier, le Père Langevin le sera jusque devant son archevêque qu'il aime comme un fils et qu'il admire comme un disciple. Pour s'excuser de ne point recevoir toutes les confidences de Mgr Taché, il écrira: " J'appartiers à une race et à une famille qui ne savent pas aduler "). ${ }^{8}$ Fier, il le sera surtout devant les puissants de la politique, devant les persécuteurs et devant les opportunistes qui laissent faire les persécuteurs. Lisez son mandement de prise de possession. De son prédécesseur il a accepté le dépôt, dira-t-il. Mais qu'entend-il par dépôt? La doctrine, sans doute, et " la pure doctrine de Jésus-Christ "; mais tout de suite et en second lieu, "le trésor si précieux de nos libertés religieuses et surtout nos droits scolaires si malheureusement foulés aux pieds ". "Comme hommes libres, comme chrétiens surtout ", ajoutera-t-il, "nous devons maintenir les droits inaliénables que la loi naturelle confère aux pères de famille pour l'éducation de leurs enfants. Au nom de ces droits sacrés sauvegardés par les traités les plus solennels, les promesses royales elles-mêmes, et reconnus par le plus haut tribunal de l'Empire britannique; que dis-je? par Sa Majesté elle-mêm€ en Conseil; au nom de la justice et de l'équité et pour l'honneur du drapeau britannique et de la noble Province de Manitoba, Nous ne cesserons de réclamer Nos écoles catholiques "). ${ }^{9}$ Le jeune archevêque prenait la un solennel engagement. Il s'y tiendra avec cette sorte d'énergie qui s'appelle magnanimité.

N'allons pourtant pas l'imaginer, comme trop souvent on l'a fait, d'une roideur orgueilleuse et cassante, prêt à mourir sous les ruines plutôt que de composer avec le possible. Au début, pendant la vacance du siège, il aurait accepté volontiers cette consigne de Mgr Taché: "Quand nous aurons perdu l'espoir d'un secours prochain

8. Lettre du 21 déc. 1893.

9. Mandement de prise de possession (19 mars 1895), p. 9-10. 
et que nous constaterons l'impuissance absolue ou le mauvais vouloir des Autorités Fédérales, alors il nous faudra bien en arriver à un arrangement qui nous permette de vivre "). ${ }^{10}$ L'histoire nous l'a appris, et sa correspondance avec le Colonel Audet en témoigne, Mgr Langevin n'a jamais refusé de négocier chaque fois qu'il y a vu le moindre espoir d'accommodement. Chaque fois, en revanche, il s'insurgera contre les restitutions à demi, contre l'aumône de mendiants à des citoyens libres. "Il ne faut ni un simple morceau de pain, ni de belles promesses, ni un arrangement boîteux ", signifie-t-il. 11 "Tout en substance ou rien ", précisera-t-il encore. "A quoi bon se contenter de lambeaux après une telle lutte ». Et pour marquer d'où s'inspire cette ferme exigence, il jette à la fin de sa lettre, ces cinq petites lignes:
"Quoi qu'il en soit de la politique, nous tenons nos droits de plus haut, et par dessus tout, nous espérons en Dieu ). ${ }^{12}$

Il disait là les hautes sources d'où procédait sa fierté. Ne lui demandez pas d'humilier ses droits d'homme et ses droits de citoyen devant le droit d'un autre, cet autre se prétendît-il de race supérieure et de droit transcendant. Contre plus fort que lui, il prend sa revanche en superbe d'âme. Un jour, c'est en 1895, il entend parler de négociations entre Ottawa et Winnipeg en vue de bâcler un règlement quelconque de la question scolaire. Il écrit au Colonel:

( Si on était sérieux à Ottawa et si on était disposé à se contenter de cela, de ce chiffon, je vous le répète, j'aurais des anathèmes foudroyants... Si on songeait d'abord à un système, comme celui d'Ontario doublé de celui d'Angleterre - écoles séparées, taxes, Ecoles normales, Livres catholiques - Nous verrions. Mais nous donner moins qu'en Angleterre! A nous des hommes libres, en face de l'Acte de Manitoba de 1870 et du jugement du Cons. Prive d'Angleterre. Allons donc! „ ${ }^{13}$

10. Lettre du 30 juillet 1884.

11. Lettre du 31 janvier 1895.

12. Lettre du 12 avril 1895.

13. Lettre du ler déc. 1895. 
Quelques jours plus tard, la rumeur se répand d'un projet de commission d'enquête sur les difficultés manitobaines, projet lancé par M. Laurier. L'archevêque y coupe court d'un mot:

(" Pas de commission, mais justice prompte et entière, tout comme s'il s'agissait d'une minorité protestante anglaise. On y gagnera à affermir la Constitution! ”) ${ }^{14}$

Apparemment, pour fonder une politique de paix ou de bonneentente, cet archevêque n'a qu'une médiocre confiance aux concessions naïves et gracieuses qui procèdent presque toujours, comme l'on sait, d'un manque de dignité et de caractère. C'est encore lui qui s'écrie: "A quoi bon des concessions faites à des enragés qui rageront davantage comme un loup qui se sent plus fort! "). ${ }^{15}$ Pour les mêmes motifs rien ne le chagrine ni ne le révolte comme les attitudes molles ou les arrondissements d'échine de ses compatriotes:

« Nous sommes les tristes victimes de l'esprit de parti et de l'avachissement des hommes au pouvoir et en dehors dans l'opposition ", gémit-il un jour. "Où est l'homme qui va nous sauver? J'agonise ici, mon cher colonel, quand je vois de près les hommes et les choses et que je me demande s'il ne me faudra pas accepter et boire jusqu'à la lie un calice d'amertume "). ${ }^{16}$

En 1912, lors de la triste affaire des écoles du Keewatin, il ne peut s'empêcher d'écrire à son ami, F.-D. Monk, alors ministre dans le cabinet Borden: ${ }^{17}$

" Mon cher ami, j’ai l'âme navrée après la lecture des dépêches de ce matin nous rapportant les raisons que tu as données pour appuyer le transfert du Keewatin sans clause pour les écoles séparées... Non seulement nos droits sont sacrifies; mais ils sont niés. J'en ai l'âme malade et je suis triste à en mourir 》.

On aperçoit quelle sève gonflée bouillonnait en cet homme, sève d'un tempérament riche, généreux à déborder, nous dirions même quelque peu volcanique. "Il faut se méfier du premier mouvement ", disait Talleyrand, " parce que c'est le bon ". Pour cette raison même,

14. Lettre du 6 déc. 1895.

15. Lettre du 14 fév. 1896 .

16. Lettre du 26 sept. 1894.

17. Lettre du 6 mars 1912. 
Mgr Langevin ne se méfie pas de sa spontanéité. Et c'est ce qui fait l'un des charmes de cette correspondance. Recueil de lettres toutes autographes, l'écriture à elle seule devient image de l'homme, écriture arrondie, mais surtout cursive. La pensée, le sentiment, dirait-on, surgissent, s'échauffent trop vite pour la plume. Et voici les demipages, des pages entières qui se bousculent sur le papier comme en bourrasque; la ligne tient parfois dans un mot, deux ou trois mots au plus, écrits d'une pointe appuyée et largement soulignés. Un jour, il décrit le vieil archevêque Taché tenant tête à ses adversaires: "Crojriez-vous que plus je le vois de près plus il grandit à mes yeux 》. Et tout de suite les exclamations se suivent, une seule par ligne, comme un quatrain:

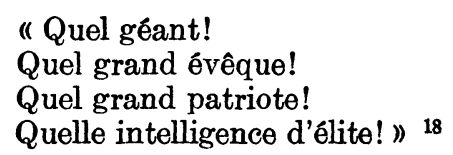

Rien de criard pourtant, rien d'emphatique en ces explosions de tempérament. En cet homme tout en droiture, sans fard ni masque, pas le moindre appui sur la chanterelle. Il a du panache, avons-nous dit, et $\mathrm{du}$ meilleur que relève souvent une pointe d'esprit chevaleresque. La 10 novembre 1895, à un moment critique pour la question scolaire, alors que ce mot de lassitude lui échappe: "Parfois je ne sais que devenir ", sa lettre se termine par cette petite phrase: "Aidez-moi à combattre. J'y laisserais ma vie volontiers ". En 1900, après tant de déboires et après qu'on l'a réduit au silence, ces autres lignes de même tonalité:

" J'ai fait mon devoir, et je devrai le payer cher même auprès des saints de Dieu; mais je puis dire comme St. Paul « Si je faisais tant que de plaire aux hommes, je ne serais pas le serviteur du Christ "). ${ }^{19}$

\section{II}

Que nous livre encore cette correspondance? On s'y attend bien: une émouvante figure d'évêque patriote. L'esprit populaire a surtout

18. Lettre du 24 janvier 1894.

19. Lettre du 16 juin 1900. 
gardé de lui cette image. Dissipons une légende. D'aucuns ont voulu faire de l'archevêque de Saint-Boniface, sinon en toutes lettres, un partisan politique, du moins un homme dont les jugements et les actes se seraient trop souvent inspirés, - fût-ce par atavisme inconscient - du sentiment partisan. Parti et nationalité, fidélité partisane et patriotisme se seraient confondus en son esprit, comme en l'esprit de tant de pauvres illuminés de son temps et de tous les temps. Il aurait accordé au parti conservateur une confiance ingénue; et, tout en servant la cause des écoles, il aurait pris soin de desservir le moins possible ses amis politiques.

Interrogeons là-dessus la correspondance. Que l'archevêque fât de tendances conservatrices comme le clergé de la province de Québec s'en payait alors la mode et pour cause, je crois qu'il n'en faut pas disconvenir. Qu'il aimât causer politique, comme tout homme qui aime suivre les affaires de son pays, le Père Langevin n'en fait nul mystère, lui qui, le 17 novembre 1892, dès sa première lettre à son ami, lui confie:

( Il m'est difficile, cette année, de faire des visites, sans cela vous me verriez de temps à autre dans votre ermitage, parce que je suis un peu comme les bonnes gens de Montréal, j'aime à entendre parler de bonne et saine politique 》.

Le colonel Audet, il faut aussi l'admettre, paraît avoir été quelque peu le professeur politique du Père Langevin. Pour remercier le Colonel de l'envoi de certains documents, le Père lui écrit: "Tout cela fait mon éducation politique " ${ }^{20} \mathrm{Et}$ comme le Colonel fréquente parfois chez M. Colin, supérieur de Saint-Sulpice, le Père estime que le vénérable sulpicien "doit être heureux d'être renseigné par un vieux médecin politique qui sait tâter le pouls du peuple et des grands. ${ }^{21}$ Admettons encore qu'en 1895 et 1896, pour régler la question manitobaine, Mgr Langevin a cru, avec beaucoup d'autres, en l'efficacité de la loi remédiatrice, bouée de sauvetage plus qu'à demi crevée, à laquelle les conservateurs aux abois avaient accroché leur sort. S'ensuit-il qu'à son acquiescement l'archevêque n'ait mis aucune condition? Écoutez-le s'en expliquer sans détours, sans ambages: si le gouvernement de Winnipeg refuse à la minorité l'octroi scolaire, l'ar-

20. Lettre du 25 aout 1893.

21. Lettre du 21 déc. 1893. 
chevêque veut à tout prix sa part de l'argent des terres; la loi " remédiatrice ne devra pas non plus 》) lier les écoles catholiques du Manitoba aux écoles publiques de la province ni aux écoles séparées de l'Ontario pour le choix des livres. "Cette clause restrictive ", appuiera-t-il, " est inadmissible... je ne dirai pas avant; je ne dirai pas après; " je suis satisfait $1{ }^{2}{ }^{2}$. Déjà le 10 nov. 1895 , il avait mis en garde ses amis trop confiants:

( Mais je crains des mutilations sous prétexte que cela aidera à faire passer la loi et que nous pourrons tout reprendre ensuite. Mais non, ce sera fini, ni. - Non, non, non. Qu'on nous rende justice, pleine justice! )

Retenons également que sa foi en la loi remédiatrice se subordonnait a une session parlementaire expéditive, tenue en 1895 et non pas renvoyée, avec la dernière imprudence, en 1896, aux dernières heures d'un parlement moribond. S'il l'accepte, même en 1896, c'est faute de mieux; et il l'accepte avec tout son entourage ecclésiastique et laỉc. Voyez comme il presse les hommes d'Ottawa:

" Que l'on comprenne bien à Ottawa que renoncer à la session sera considéré comme une trahison, ou, au moins, une lâcheté maladroite. Comment pourrions-nous décemment lever le doigt en faveur de ce Gouvernement qui, après avoir évité de faire un pas pour nous secourir, ira demander à l'électorat de le confirmer au pouvoir? Comment pourrons-nous même nous défendre de déclarer hautement que cette conduite nous révolte? ") ${ }^{23}$

Quelques mois plus tard, atterré par les hésitations d'Ottawa qui balance entre la tenue d'une session et une reprise des négociations avec Winnipeg, la vigie de Saint-Boniface lance cet autre avertissement quasi prophétique:

« Si le Gouvernement veut vivre qu'il se décide à une session d'automne, s'il refuse, c'est qu'il est atteint de la folie qui annonce la fin d'un pouvoir. Une session d'automne ou la mort. Si

22. Lettre du 14 fév. 1896. Incidemment l'on apprend, en cette lettre, les noms de ceux qui ont préparé la loi réparatrice ou du moins l'ébauche de cette loi: « Ewart croit qu'il faudra d'abord sommer le Gouv. Greenway de passer cette loi appelée - Act for separate schools " et amené comme a way of supplementing la loi de 1890. C'est Ew ak', Bernier, Larivière, M. Cherrier, les juges Dubuc et Prud'hommd, et un peu Prendergast qui y ont travaillé. On m'a rendu compte ". (Lettre du 21 fév. 1895.)

23. Lettre du 12 fév. 1895. 
on décidait des élections générales je serais le premier à crier au Pays tout entier - ( On nous a trahis! ) ${ }^{24}$

Ceux qui ont lu, dans la livraison de septembre 1947 de la Revue d'Histoire de l'A mérique franŁaise, les extraits que nous y avons publiés, de lacorrespondance L ANGE VIN-AUDET savent, en quelle liberté d'esprit l'archevêque jugeait le parti conservateur et ses chefs. Et ces jugements d'homme libre, on les rencontre, avons-nous dit, non seulement sous la plume de l'archevêque trompé et désabusé; le Père Langevin les énonce aussi fermement. Lisez, par exemple, cet extrait d'une lettre du 21 décembre 1893 où le Père s'affranchit assez carrément des façons de voir de son professeur politique:

« Je voudrais bien partager votre confiance dans le parti conservateur tel qu'il est gouverné, mais cela m'est impossible. On tergiverse à Ottawa, on veut éviter l'ennui et le danger de mettre les Chambres en mesure de se prononcer en faveur ou contre la minorité de Manitoba. Un gouvernement qui ne protège plus le faible n'a plus sa raison d'être. Que gagne-t-il à attendre? )

"Que fait Thompson?"...

a Assurément, je crois bien que le parti conservateur renferme seul les éléments de vie et de prospérité nationale, mais où sont ses chefs? Il est acéphale ou il a des tentacules de pieuvre pour têtes... )

Ces jugements, comme la sévérité s'en accroîtra, quand les tergiversations et les divisions intestines du cabinet d'Ottawa auront fourvoyé dans une impasse la question scolaire:

"Si à Ottawa) - écrit alors l'archevêque - (le cabinet ne peut pas s'entendre, alors ils sont pris de la folie qui présage la

24. Lettre du 27 sept. 1895.- - Des libéraux éminents n'auraient-ils pas regretté leur conduite da 189.3 - je vaux dire, leur obstruction parlementaire d'alors à la loi remédiatrice? En 1928 je passai quelques semaines à Saint-Boniface, Man. Pour un cours en préparation sur l'histoire des écoles des minorités, j'y étais allé consulter les archives de l'Archevêché. Je me ménageai aussi de longs entretiens avec les survivants de la lutte scolaire manitobaine. Je vis plusieurs fois, entre autres, M. le juge James Prendergast, ancien député de Winnipeg. Voici ce que je trouve dans mes notes recueillies à cette date:

a S. Boniface, Man. 5 juin 1928.

Recueilli, ce soir, de la bouche du juge James Prendergast: "Un jour, c'était pendant la guerre, le juge Brodeur [ancien ministre du Cabinet Laurier] me dit: auge, nous nous sommes trompés en 1896 en nous opposant au bill remédiateur. Nous aurions dâ l'appuyer pour les principes qu'il incluait $\rrbracket$. Et M. Prendergast d'ajouter: "Plus tard, ce fut aussi ma conviction ". L.G. 
ruine finale. Je réserve pour le cabinet Bowell des anathèmes qui ne sont pas de paille. Ces messieurs font bien de ne pas traiter officiellement avec moi, de ne me rien communiquer directement. Laissez-les faire de grâce, ils me fortifient. J'aurai les mains libres pour frapper ). ${ }^{25}$

Mêmes jugements et sans plus d'aménité, à l'adresse des partisans aveugles:

( Les attaques du " Moniteur ) (de Lévis) et du " Quotidien 》 me prouvent que les partisans quand même du côté des Conservateurs se fichent des évêques comme les pires libéraux euxmêmes. Celam'instruitetme rend plus libre d'agir au besoin ). ${ }^{26}$

Mais, se demandera-t-on, quels sentiments cetarchevêque, dégoôté des conservateurs, entretient-il à l'égard du parti libéral ? Un premier fait s'impose, souligné d'ailleurs par toute la correspondance: Mgr Langevin juge hommes et partis politiques selon leur attitude à l'égard de Ja question scolaire. Pour lui c'est le mètre souverain et qui n'implique rien ni de si factice ni de si injuste. Selon qu'ils se comportent à l'égarddu droit minoritaire, l'archevêque mesure le courage des hommes, leur sagacité politique, leur respect du droit, de la constitution du pays, leur pensée de fond sur la Confédération. Selon ces normes, le parti libéral ne pouvait guère s'attendre à beaucoup de complaisance. Il portait un passé assez chargé d'anticléricalisme. Sa presse malmenait volontiers l'archevêque. Elle l'accusait de faire le jeu des conservateurs, et surtout de compromettre, par son intransigeance, les droits de la minorité. En outre le parti libéral se donnait l'air de louvoyer, d'éviter tout engagement précis. Disons-le tout net: Mgr Langevin n'éprouve que méfiance pour le parti libéral de l'époque et pour son aile québécoise. Celle-ci lui parait faire plus que bon marché du droit minoritaire. Dans cette méfiance, a-t-il enveloppé, avant et après 1896, le chef de l'opposition: Wilfrid Laurier? On pourra s'étonner, si l'on veut, mais les premiers jugements de l'archevêque sur cet homme politique ne s'inspirent d'aucune hostilité systématique: A son ami le Colonel qui taxe M. Laurier de lâcheté, il riposte, le 26 septembre 1894: "Avouez que si Laurier est lâche et perfide, les chefs du parti

25. Lettre du ler déc. 1895.

26. Lettre du 18 août 1895. 
conservateur ne sont point braves ". Un peu plus tard, en 1895, à la recherche d'un sauveur, l'archevêque écrit:

"Chapleau serait-il l'homme? Je n'en vois pas d'autre. Si le cour ne lui dit rien en face de telles éventualités, c'est un misérable qui sera maudit le jour où il n'arrivera qu'après le naufrage ou après la victoire pour la curée. Pas un seul homme ne se présentera donc? Laurier seul semble être quelque chose malgré ses phases lunaires. ") ${ }^{27}$

Plus tard, à la veille du débat sur la loi réparatrice, il voudra qu'on évite d'embarrasser le chef de l'opposition. Même si l'homme reste pour lui l'indéchiffrable énigme, il ne se défend pas d'espérer:

"Laurier! que fera Laurier?"

(Il ne faudrait pas le provoquer, afin de lui laisser une issue pour revenir au bon sens. Il abandonne l'idée d'une commission, m'écrit quelqu'un qui l'a vu privément... Sir Charles [Tupper] s'affirme comme un protestant sincère et zélé. $M$. Laurier a-t-il peur de s'affirmer comme catholique! Les Grits d'Ontario lui en seront-ils reconnaissants! Vous le dites bien, l'anglais ne cède que devant le coup de poing et l'intérêt! Laurier va-t-il jouer à la Papineau?" " ${ }^{28}$

Enfin, plus tard, le 9 nov. 1898, - je trouve copie de cette lettre dans la correspondance Langevin-Audet - Mgr Langevin, tout en y mettant des nuances, reconnaîtra loyalement les bons offices de Wilfrid Laurier devenu premier ministre:

" Nous avons accepté volontiers les concessions qui nous ont été faites à la suite des luttes du passé et grâce à l'heureuse influence et au bon vouloir que vous avez bien voulu montrer, la condition de nos écoles est certainement améliorée... ")

( Mais je me hâte d'ajouter, Monsieur le Ministre, que j'ai le ferme espoir de voir arriver le moment où nous réussirons à régler les difficultés présentes et à assurer la paix, car j'ai pu constater en plusieurs circonstances chez ceux qui tiennent en mains le règlement de cette question difficile, des disposi-

27. Lettre du 1er déc. 1895. Ajoutons ici en note que Mgr Langevin ne se faisait aucune illusion sur Chapleau. Il écrit, par exemple, le 14 fév. 1896: "Il faudrait un homme supérieur et un homme de principes pcur représenter le parti dans Québec. Chapleau le fortifiera politiquement, mais ce n'est pas lui qui le consolidera au point de vue doctrinal ").

28. Lettre du 14 fév. 1896. 
tions excellentes et un désir sérieux d'arriver à une conclusion, et vous me permettrez de compter encore sur votre présent appui ».

Pas plus que son indépendance politique, le patriotisme de $\mathrm{Mgr}$ Langevin ne nous semble donc discutable. Ce patriotisme, tenteronsnous de le définir, d'en marquer la note dominante? Les textes nous apportent moins une définition qu'un état d'esprit. S'il fallait risquer des précisions, peut-être pourrait-on parler de patriotisme de temps de crise, patriotisme inquiet, teinté d'un peu de pessimisme. Ainsi le veulent, hélas, l'époque, le spectacle de la vie nationale et politique, l'espèce de tragédie où l'archevêque dut assumer l'un des grands rôles. L'homme avait trop de cœur et l'avait trop haut pour ne pas beaucoup souffrir des misères morales de son temps. Loin, très loin là-bas dans l'ouest, entouré d'une très petite garde, pas toujours unie, obligé de compter sur un Québec toujours divisé par l'éternelle et petite politique, il ne peut se défendre d'une douloureuse impression d'isolement et d'abandon. Il ne pardonnera jamais aux gens de sa race d'avoir perdu la bataille scolaire par leur désunion et leur manque de sens politique.

A-t-il jamais confessé, comme on le lui a fait dire parfois, qu'avant son arrivée dans l'ouest, anesthésjé comme tant d'autres par la molle et quiète atmosphère de la vieille province de Québec, il ignorait tout du patriotisme? Si oui, convenons que le noble sentiment s'éveilla tôt dans le cœur du Père Langevin, et tout de suite, avec cette pointe d'inquiète souffrance qui perce presque toujours. Car c'est bien lui qui, un an à peine après son arrivée au Manitoba, écrit des confidences comme celle-ci:

« Je n'ai jamais désespéré, mais l'angoisse me serre le cœur parfois... O! si tous avaient cette foi nationale au cœur... Assurément tout est providentiel, chez nous, et il $[y]$ aurait une insigne ingratitude à le nier. Mais le Maître des rois et des peuples, le Christ qui aime les Franes ne demande-t-il pas de notre part une action plus prononcée? Nous ne vivrons pas toujours de miracles $).{ }^{29}$

Foi en la Providence, en la mission sacréede sa race, mais, en même temps incertitude, doute, anxiété, devant le terrible présent, devant

29. Lettre du 25 oct. 1893. 
l'insouciance d'un peuple qui joue avec sa vie, son avenir, c'est tout le drame qui étreint ce cœur d'homme.

Les premiers échecs de la minorité manitobaine devant les tribunaux et à Ottawa, jettent le désarroi dans l'âme du curé de SainteMarie de Winnipeg:

"Oui je suis malade au moral! Où allons-nous? Pas de dもsaveu de la loi des Écoles au Nord-Ouest. Mgr l'espérait 1). ${ }^{30}$

Deux mois plus tard, encore semblables propos:

"Où est le sens de la justice chez les Anglais et l'esprit catholique et national chez les Canadiens français? La situation est peut-être une des plus graves de notre histoire. Jamais nous n'avons été aussi près de l'abîme. Est-ce l'aurore d'un grand triomphe, j'espère et j'ai foi en l'avenir de mon pays et de ma race parce que je crois à une mission divine; mais je me demande d'où va venir le salut? Que les saints du Canada aient compassion de nous! \) ${ }^{31}$

Cette amertume, il faudrait peut-être dire cette angoisse, ne fera que grandir quand le curé de Sainte-Marie de Winnipeg, devenu archevêque de Saint-Boniface, se verra chargé de l'écrasante croix. Lourde succession, en effet, que celle du vieil archevêque Taché. Suite ordinaire de tous les règnes prolongés, bien des choses qu'on aurait pu croire solides, s'écroulajent tout a coup en dissolution. Le nouvel archevêque le note mélancoliquement:

" Mais qu'il me faut de patience et de prudence pour tenir debout cette succession qui se disloque de toutes parts sans que les gens s'en doutent! Le grand homme ne tenait qu'une partie dans sa main, le reste qu'il croyait tenir attendait le coup de la mort pour s'émanciper "). ${ }^{32}$

Ce chef de minorité, pris entre le fanatisme d'ennemis implacables et la lâcheté des siens, gravit véritablement un calvaire. Le mot " agonie " revient dans ses lettres, avec un peu d'excès. Entendez-le:

30. Lettre du 24 janv. 1894.

31. Lettre du 20 mars 1894.

32. Lettre du 4 sept. 1895. 
(J'Étouffe parfois! O! agonie! ) ${ }^{33}$

N La fatigue commence à me dominer. Mes sollicitudes me font souffrir l'agonie d'un crucifie $).{ }^{34}$

a 0 ! mon pays, mon pays! Pour la première fois dans ton histoire, tu ne peux pas même trouver un homme! $)^{35}$

a Vous flétrissez bien la cohue égoiste des nôtres!! C'est écoeurant. ${ }^{36}$

" Oh! qu'il faut avoir de la foi et aimer son pays de tout son cœur pour ne pas désespérer. J'agonize. ॥) ${ }^{37}$

Huit ans plus tard, vaincu, désarmé, réduit au silence, sa blessure reste toujours vive, grande ouverte:

« Tout est perdu fors un léger bris de droits scolaires que la première brise de fanatisme peut nous enlever, et l'honneur sauf, mais pas chez tous! ”

"Où va le peuple canadien avec ces reculades encensées par les politiciens et les journaux à la solde de sir W. et de la Franc-Mą̧onnerie ?... ).

( Je suis triste jusqu'à la mort. Quelle agonie j'éprouve comme archevêque et comme patriote! Quel dégoût me soulève le cœur! ) ${ }^{38}$

On note pourtant, dans cette âme aux ressorts vigoureux, de promptes et fréquentes ressaisies, et ce, au lendemain des jours les

33. Lettre du 4 sept. 1895.

34. Lettre du 8 oct. 1895.

34. Lettre du 1er déc. 1895.

36. Lettre du 14 fév. 1896.

37. Lettre du 14 déc. 1897.

38. Lettre du 19 avril 1905. 
plus sombres. Le 18 aoat 1895, il termine ainsi une lettre trop chargée de mauvais pressentiments:

( J'espère quand même. Il suffit de quelques hommes de cœur pour nous sauver. Le Bon Dieu nous les donnera. J'espère ").

En 1903, alors que Mgr Bruchési, de retour de Rome, lui rapporte des paroles d'espoir, il écrit aussitôt:

« La déclaration de Mgr Bruchési ravive toutes mes espérances $)$.

" J'ai tant crié dans le désert! »)

"J'ai été si abandonné!)" 39

Est-il besoin de dire que ce patriotisme est avant tout d'essence canadienne-française? Devenu homme de l'ouest, et très attaché à son nouveau pays, Mgr Langevin reste Canadien français; il n'oublie jamais que, par naissance et culture, il est fils du Québec. Après la foi catholique, c'est pour l'héritage culturel des siens, pour la langue, les traditions originelles, qu'il livre la grande bataille. C'est dans ses droits, sa dignité de Canadien français que, tout particulièrement, les lois spoliatrices de l'Ouest l'atteignent et l'exaspèrent. En 1894, la Free Press de Winnipeg, "journal vendu à Greenway et au C.P.R. ", publie un article perfide, intitule: Put the saddle on the other horse. Le journal prétend démontrer qu'après tout les catholiques du Manitoba ne sont pas plus maltraités que les protestants de la province de Québec. Aussitôt le curé de Sainte-Marie de Winnipeg alerte son ami Audet et le prie de l'aider à confondre la thèse malhonnête. "Il s'agit de l'honneur de Québec ", écrit-il. ${ }^{\circ}{ }^{\circ}$ Il suit de très près le mouvement des idées dans la vieille province, aux aguets des moindres signes d'éveil national, de toute précision des idéaux nationaux. C'est l'époque, hélas, où, en cette matière, l'on n'est guère prodigue de clartés, de définitions nettes. En 1903, il accuse réception de la brochure de M. Henri Bourassa: Le patriotisme canadien-français, ce qu'il est, - ce qu'il doit être, en ces termes:

" La brochure de M. Henri Bourassa sur les Canadiens Fransais m'a fait grand plaisir. C'est bien cela "). ${ }^{4}$

39. Lettre du 3 fév. 1903.

40. Lettre du 15 fév. et du 16 avril 1894.

41. Lettre du 2 fév. 1903. 
Noterons-nous, à l'honneur de cet archevêque d'origine québécoise, une largeur de vue qui ne s'enferme pas dans les frontières du provincialisme. Il eût souhaité, sans doute, peupler l'Ouest de gens de sa race et de croyants catholiques. Et qui pourrait l'en blâmer? Il écrit, presque au lendemain de son arrivée là-bas:

" Le pays que je parcours a été visité par nos pères; nous y avons le droit de premier occupant. Si le Gouvernement avait chargé des sociétés (dirigées par des laïques et des prêtres), de coloniser le Pays, il y a 20 ans, nous aurions ici un demimillion de Canadiens-français vivant comme des princes "). " Il est encore temps de faire quelque chose, e'est-à-dire, de semer cette mer d'herbe des ilots de nos gens. Dans 50 ans, comme après la conquête du Canada, les Anglais se réveilleront tout étonnés aux bruits d'une armée de French chantant "Vive la Canadienne ). ${ }^{42}$

Pour immigrants, il eât voulu: « Des cultivateurs! Des cultivateurs! Des paysans de Normandie, de Bretagne et des croyants ". 43 Mais ces croyants, il ne prétend point les recruter uniquement chez les siens. Il eût souhaité une "Société de colonisation du Manitoba et $\mathrm{du}$ Nord-Ouest ayant trois ou quatre sections, francaise, belge, ainsi que canadienne et irlandaise $1{ }^{4}{ }^{4}$ En 1899 il entreprend de fonder a Saint-Boniface une " bibliothèque canadienne ") où, à côté des revues et des ouvrages d'auteurs canadiens, prendront place aussi les ouvrages publiés par les étrangers sur le Canada. Et son projet s'inspire de cette pensée: " afin qu'il y ait, au moins, de l'autre côté de la hauteur des terres, un endroit où l'on pourra trouver matière à s'instruire sur notre pays $1{ }^{45}$

\section{III}

En cette correspondance d'évêque, l'on s'attend à rencontrer l'évêque. Il y est et pour grandir l'homme et le patriote. Envoyé dans l'Ouest, selon toute apparence, pour se préparer à la succession de Mgr Taché, il nous plaît tout d'abord que le Père Langevin ait eu

42. Lettre du 25 oct. 1893.

43. Lettre du 15 nov. 1893.

44. Lettre du 19 aoat 1893.

45. Lettre du 30 oct. 1899. 
assez de foi et d'esprit pour ne pas désirer l'épiscopat. Sans doute, a-t-il peu goaté une pétition du clergé séculier qui eât voulu pour chef l'un des siens. Très attaché à sa communauté, l'Oblat estime peu gracieuse la démarche des séculiers:

" Nous, oblats, nous sommes menacés d'ostracisme, sans dou te pour nous récompenser d'avoir donné un évêque incomparable au nord-ouest canadien! /) ${ }^{46}$

Le Père général de la communauté qualifiera la démarche de " triste affaire ", de "grand acte d'ingratitude ". Mais si le curé de Ste-Marie de Winnipeg désire un Oblat sur le siège de Saint-Boniface, il ne s'ensuit point que cet Oblat doive être le Père Langevin. D'ailleurs, observe le curé de Sainte-Marie, le doyen des suffragants de Saint-Boniface, Mgr Grandin, " n'a pas voulu se donner l'air de trop insister (à Rome) pour un des nôtres ». Et quant au Père général, il a déclaré: "Pour moi, je me tiens neutre... je dois me contenter de l'action des suffragants, et si Rome nomme un apôtre séculier, j'en suis déjà tout consolé; seulement je crois qu'il est de notre devoir de respecter jusque-là le vœu, la volonté du vénérable défunt... / ${ }^{47}$

En toute vérité la succession de l'archevêque Taché fait peur au Père Langevin. Le 12 juillet 1893, il s'en ouvre à son ami le colonel:

( Je ne suis que vicaire de missions à Winnipeg, j'espère échapper au reste, car vous ne sauriez croire comme la seule pensée d'une succession gigantesque m'écrase. Personne ne pourra me persuader que je suis de taille à porter cette cuirasse ). (Avertat Deus! " 48

A une " exhortation chaleureuse " de son ami " d'accepter le fardeau ", il répond:

«Oui, certes, je veux boire ma bonne part du calice amerJe sais bien que le petit David a fait merveille avec sa fronde parce que le Bon Dieu l'a aidé; mais voulez-vous donc que je m'attende à un miracle? ") ${ }^{49}$

46. Lettre du 6 et 30 juillet 1894.

47. Lettre du 30 juillet 1894.

48. Lettre du 12 juillet 1894.

49 Lettre du 2 aoat 1893. 
Le 6 juillet 1894, le Père Langevin peut encore se rendre ce noble témoignage:

« Ma grande force est de n'avoir jamais dit un mot, fait un pas, l'ombre d'une démarche vers ce Calvaire, non, certes, par lâcheté; mais par conscience. Trois jours avant le grand deuil j'aurais pu presser la chose. Mgr Grandin m'ayant consulté ad hoc, je lui dis: "Attendez qu'il vous en parle ». Mgr d'Ottawa m'a dit que s'il avait su cela, il aurait pressé Mgr Grandin de tout régler avant le grand dénouement. Mais, je vous le demande, m'appartient-il de dire un mot en ce sens? )

" Et puis, il peut se faire que l'œuvre de Mgr Taché soit mieux sauvegardée, continuée par un séculier "). ${ }^{50}$

Nommé officiellement par Rome le 8 janvier 1895, cet évêque sera un évêque avant tout, c'est-à-dire un chef spirituel. Toute la correspondance accuse fortement ce trait par quelques lignes, quelques mots jetés ici et là dans l'abandon de la confidence, quand ce n'est pas en de plus larges développements. Dans la lutte scolaire, le noble champion voit au-dessus de tout, le combat pour l'Église.

" Oui, nous devons nous grouper tous autour du drapeau qui symbolise nos droits, nos libertés: c'est le drapeau de la Ste-Église! ") ${ }^{51}$

Délaissé, oublié là-bas, dans une défaite du droit minoritaire qui est surtout sá propre défaite, il laisse tomber sur le papier ce beau cri d'homme de foi:

(Qu'il est doux de travailler pour Dieu seul... ) ${ }^{52}$

En 1912, il accepte le compromis Roblin. Tout aussitôt la presse libérale lui reproche de courtiser les conservateurs. Sa correspondance s'enrichit alors de ce commentaire où s'affirme, une fois de plus, devant les politiciens, son indépendance d'homme d'Église:

( En 1898 en voulant aider mes enfants des campagnes, et, a la demande du Pape, en tirant parti du soi-disant règlement Laurier-Greenway, j'ai été blâme par les "Conservateurs 》; aujourd'hui, en voulant tirer parti de la situation qui m'est

50. Lettre du 6 juillet 1894.

51. Lettre du 16 juin 1900.

52. Lettre du 17 mars 1903. 
faite a Ottawa, et a Winnipeg, je parais vouloir aider les "conservateurs ". Tant mieux pour les deux! Mais je fais mon devoir d'archevêque et voilà tout! 》) ${ }^{53}$

On le sait: rien comme l'épreuve pour donner la mesure de l'âme humaine. La souffrance ramène l'homme à son exacte taille. Sa grandeur d'âme, l'évêque la révéla surtout, en 1908, à l'heure où Rome imposa silence au champion de la minorite. Dans la vie de cet homme ardent, si sûr de son droit, de tempérament si fier, ce fut, croyons-nous, l'épisode tragique, l'épreuve suprême. Surpris, humilié, à la face et à la grande joie des opportunistes et des persécuteurs, l'archevêque s'inclina dans l'obéissance. Rome avait parlé. Sous Mgr Taché la coutume s'était établie, dans les églises manitobaines, d'écouter debout, comme pour l'évangile, la lecture des documents pontificaux. ${ }^{54}$ L'archevêque Langevin traita, avec le même respect, la consigne de Léon XIII. Au souvenir de ces heures douloureuses, il Ǵcrira:

" J'ai beaucoup souffert, mais j'ai obøi, je suis en paix ". ${ }^{55}$

Et encore:

( Si Rome avait voulu, j'aurais fait davantage Mais vous savez ce que discipline veut dire dans un régiment bien formé. ) ${ }^{56}$

Évêque catholique, l'on ne sera pas étonné que l'archevêque de Saint-Boniface se soit montré un grand évêque canadien, en ce sens que, dans son amour de l'Église, il enveloppa toute l'Église canadienne. A sa défense du droit scolaire il fit ce qu'il put pour rallier l'appui de tout l'épiscopat anglo-canadien:

" Je vais m'assurer l'appui formel de l'épiscopat anglais que je compte comme très sympathique à notre cause et au mois de janvier il y aura du tremblement $).{ }^{57}$

53. Lettre du 31 mars 1912.

54. Voir le Mandement de prise de possession, p. 6.

55. Lettre du 2 fév. 1903.

56. Lettre du 19 avril 1905.

57. Lettre du 2 aout 1895. 
A l'automne de 1895 il dépêche le Père Lacombe, o.m.i. à ses collègues de langue anglaise. Il en donne la nouvelle au Colonel Audet:

« Le Père Lacombe vous verra; il est charge de la mission secrète d'aller voir l'episcopat d'Ontario et de Québec au sujet des écoles ). ${ }^{58}$

Disciple de l'archevêque Taché, il avait retenu, des écrits de son prédécesseur, une leçon qui l'avait particulièrement frappé:

(Il exprime quelque part ) - (écrivait en ce temps-là, le Père Langevin) (" une pensée si large, au sujet de l'union du clergé canadien de l'Atlantique au Pacifique, il montre si bien qu'il faut enfin sortir de l'ornière diocésaine et s'intéresser au Pays tout entier, que j'en ai été ravi et grandement consolé ).. ${ }^{59}$

Enfin, pour auréoler, et de façon admirable, ce nous semble, cette physionomie d'évêque, nous verrons en lui l'un des précurseurs de l'action catholique entendue dans un large sens, dans un sens plus large qu'on ne l'entend trop souvent. Nul peut-être, et il y avait quelque mérite en son temps, nul n'a mieux̃ admis, compris, prêché le rôle des laïcs dans la vie de l'Église et dans la vie publique. Nous dirions même: qui a cru plus que lui, par exemple, en l'efficacité et en la nécessité du journal, journal laïc, journal d'idées et journal de combat? Dans sa correspondance avec le Colonel il y revient je ne sais combien de fois. A propos d'un mouvement d'immigration catholique vers l'oucst, le Père Langevin écrit à son ami le 19 août 1893: "Si vous aviez votre journal!") Quelques mois plus tard, à propos d'une regénération de la politique canadienne, le Père revient encore à son idée: " Mais pour cela il faudrait un homme et un journal. Est-ce que cela ne vous tente point? " ${ }^{60}$ En 1909 il garde toujours son rêve:

" Le monde marche par les idées, et les semeurs de bonnes idées sont ses plus grands bienfaiteurs "). " Mais il faudrait un journal orienté dans ce sens et qui poursuivrait son but à travers tous les obstacles. 》)

58. Lettre du 1er déc. 1895.

59. Lettre du 28 nov. 1893.

60. Lettre du 15 nov. 1893. 
A l'époque il salue le projet du DEVOIR comme une réalisation possible de son rêve. Et il précise, à sa façon pittoresque, le rôle fécond du journal catholique:

« Vous m'étonnez beaucoup en me disant gu'un journal catholique n'est pas nécessaire. C'est pourtant le meilleur semeur d'idées et il y a entre le travail du journal et celui du particulier la différence énorme qui existe entre une semeuse automatique allant vite et profond et la main de l'ancien semeur à bras avec sa poche sur le dos! $\nu^{61}$

Les laïcs, il leur taille bien d'autres besognes que celle de journaliste. Ses lettres se font éloquentes, pressantes, pour les inciter à prendre leurs responsabilités de catholiques et de citoyens, pour leur assigner leur vrai rôle, rôle de premier plan, dans la défense du droit, des minorités, de la constitution du pays. Sa pensée ne craint point, sur ce sujet, les formules catégoriques:

a Il n'y a pas de puissance sous le soleil qui puisse dispenser les catholiques canadiens de défendre la constitution de leur pays outragée dans ce qu'elle a de plus sacré 1$){ }^{62}$

Ce combatif ne peut souffrir les opportunistes et les timides qui attendent toujours, pour agir, un signe du curé ou de l'evêque, qui n'aiment rien tant que s'abriter derrière une soutane. Pour flageller ces pusillanimes, il trouve des propos énergiques et savoureux:

"Que vous êtes done sage, archisage en disant qu'il ne faut s'abriter derrière l'épiscopat ! C'est le hic. Allez done, allez toujours! Les fideles sont forts du droit naturel et de la constitution! Qui peut les arrêter!... J'ai crié cela tout haut cent fois ".

"Mais on dirait qu'il faut compromettre le clerge a tout prix ").

"On dirait que le Canadien n'est catholique que lorsqu'il tient le prêtre par la soutane; et quand il ne la tient plus, il croit qu'il doit nécessairement sacrer le prêtre... Allez donc, fidèles. Vous avez vos devoirs de citoyens d'un pays libre et ce n'est pas quand la constitution baśe sur le droit naturel vous dit qu'elle est violese et qu'elle souffre violence qu'il faut

61. Lettres du 17 janvier et du 23 mars 1909.

62. Lettre du 16 juin 1900 . 
aller de jour ou le soir au presbytère ou à l'évêché pour demander ce qu'il faut faire!... )

" Oh! si j'avais été dans les rangs des fidèles, que j'aurais ferraillé de bon cœur, affectant même de ne pas trop m'occuper du clergé »).

" J'ai entendu un évêque dire à Mgr Merry del Val » [Cet évêque ne serait-il pas Mgr Langevin?] - Nous ne pouvons pas nous évêques abdiquer notre titre de citoyens et fouler aux pieds ou laisser violer la constitution de notre pays quand elle proclame le droit naturel des parents $).{ }^{63}$

Souvent il se prend à souhaiter, dans la politique canadienne, la présence d'un groupe de députés indépendants. Un jour que son ami Audet lui a parlé de la naissance possible d'un groupe de cette nature, il exulte:

" Merci de ce que vous me dites au sujet du parti et de ses hommes. Cela me console et m'encourage beaucoup. Rallier les forces vives, tracer un programme sur des bases solides, chrétiennes; lancer la pierre à Québec et la relancer à Ottawa; et travailler à éloigner les malheureux qui conduisent le Pays à la ruine. Deo gratias! Il y a donc encore des hommes dans notre beau pays. ) 64

C'est l'évêque, du reste, qui dans une lettre antérieure, avait jeté l'idée à son ami:

" Tous les Canadiens devraient s'unir et imposer un programme, former un parti qui balancerait le pouvoir et que les Anglais auraient intérêt à ménager. Nous sommes trop esclaves des Anglais par esprit de partisannerie ). ${ }^{65}$

" 20 députés bien unis ) écrira-t-il encore, " et arborant ce simple mot "Constitution " feraient des merveilles ". ${ }^{66}$

Dans l'intervalle, dégouté des vieux partis et surtout de la politique des siens, il avait même souri quelque peu au projet d'un centre catholique:

(" Je crois a l'existence de bons eléments de succès; mais qui va les rallier? "

63. Lettre du 27 avril 1903.

64. Lettre du 15 oct. 1894.

65. Lettre du 7 juin 1894.

66. Lettre du 27 avril 1903 
(" Voilà ce qui me porte à croire qu'un parti-centre ou une ligue catholique est notre unique moyen d'échapper aux griffes des rouges, au baiser hypocrite des Conservateurs sans conscience, des Judas, et de sauver notre pays qui n'a jamais été plus malade. Notre pire ennemi, c'est nous! Nous sommes encore assez forts pour dicter notre volonté à la Confédération entière $).{ }^{67}$

On aperçoit l'intérêt de cette correspondance Langevin-Audet. Elle accuse, en les éclairant mieux, quelques-uns des traits déjà connus d'une passionnante figure d'évêque. Homme discuté sans doute, comme tous ceux qui ont courtisé leur conscience plus que leur intérêt et qui ont voulu faire quelque chose de leur vie; mais homme de cœur et de foi qui dépasse tellement ceux qui l'ont décrié. Tous les esprits libres qui, non sans mérite souvent, se refusent à l'admiration des idoles creusés et des fausses gloires, peuvent, pour une fois, admirer presque pleinement. Pourquoi faut-il qu'un préjugé, en partie fondé et entretenu par l'histoire, n'admette la grandeur humaine, dans ses larges dimensions, que chez les personnages de haute volee qui vivent leur vie sur un thếtre vaste et pompeux? Serait-ce que, pour sortir de l'ordinaire mesure, l'homme ne se puisse passer de ce décor ou que, pour relever la taille de l'acteur, il faille l'ampleur et le prestige de la scène? L'histoire néglige ou rapetisse par trop, parce que moins facile à découvrir, l'héroïsme des petits, cet humus obscur des vertus sans apparat où se fondent pourtant les assises morales d'un pays. La grandeur humaine est chose du dedans et non du dehors. Et si elle se mesure exactement aux dimensions immatérielles de l'âme, il importe peu que l'archevêque Langevin n'ait été que le chef d'un petit peuple de quelque 50,000 âmes, à demi perdu dans le far west. En ce champion chevaleresque du droit - du droit qui a partout même dignité et même taille - il faut voir, non seulement un des plus nobles fils du Canada, mais, je n'hésite pas à l'écrire, une des grandes âmes de son temps.

Lionel Groulx, ptre.

67. Lettre du 14 déc. 1897. 